\title{
Ironie als Verführungskunst: Kommunikative Funktionen und pragmatische Effekte ironischer Äußerungen zur Schaffung von Gruppenbildung in halbstrukturierten Gesprächen
}

\author{
Nicolò Calpestrati (Milano)
}

\begin{abstract}
Irony is a double-edged sword. It is a rhetorical strategy, which in spoken language is mostly used for entertainment, but can also be used as a face threatening act.

In semi-structured conversations, such as talk show rounds, the effects of irony depend on variables such as the type of TV talk shows in which the conversation develops, the participants involved and the relationships between them.

This article deals with the identification of the communicative functions of ironic utterances, how they are handled by the various parties involved (guests and TV-presenters, the public in the studio and the audience at home) and their pragmatic effects on the development of the ongoing communication.

Five episodes of the NDR talk show form the corpus of this work. The observation of both linguistic and paralinguistic markers reveals irony as a rhetorical strategy which, in the context examined, has a mainly seductive function. In fact, it is used from speakers to catch the hearers' attention, creating affiliation and alignment. This effect is realized through the management of the relationships between interlocutors who belong to different communication circles and are involved in communication in different ways.
\end{abstract}

\section{$1 \quad$ Einleitung}

Ironie gehört zu den Techniken des Komischen (cf. Dynel 2009: 1289; Norrick 2009: 261; Calpestrati 2020a: 136), die sowohl in mündlicher als auch in schriftlicher Kommunikation mit mindestens zwei Interagierenden und einer Zielperson (auch: Target) auftritt, an die sich die ironische Kommentierung richtet (im Falle der Selbstironie fällt sie mit dem Sprecher selbst zusammen), z. B. die Aussage „schönes Wetter!“, die von A geäußert und an einem stürmischen 
Tag an B gerichtet wird. Ironie ist in verschiedenen Disziplinen, von der Rhetorik bis zur Philosophie, ${ }^{1}$ von der Linguistik bis zur Kognitionswissenschaft und Psychologie ${ }^{2}$ untersucht worden. Zur Analyse und Beschreibung ihrer Funktionen wurden unterschiedliche Ansätze angewendet, aber einige von ihnen weisen Beschränkungen auf (cf. Colston/Gibbs 2007: 4). Einige Studien befassen sich nämlich mit der Analyse von ad-hoc erstellten Beispielen bzw. Daten, die aus dem ursprünglichen kommunikativen Kontext herausgelöst wurden und daher nicht zeigen können, wie Ironie sich tatsächlich entwickelt und wie mit ihr in einem realen kommunikativen Rahmen umgegangen wird. Ironiefunktionen sind in verschiedenen kommunikativen Kontexten untersucht worden, seien es fiktive (z. B. ein Witz) oder reale (z. B. ein spontanes Gespräch); es scheint aber, dass bis heute solchen kommunikativen Kontexten, die eine Mischung realer und fiktiver Elemente aufweisen, wie die Talkshow-Dialoge, zu wenig Aufmerksamkeit geschenkt worden ist: Es handelt sich grundsätzlich um ein Genre, in dem Ironie eine Rolle spielt. Charakteristisch für Talkshows ${ }^{3}$ ist die Unterhaltungsfunktion (cf. Burger 2005: 23), die nicht nur mit der Art der vermittelten Inhalte zu tun hat, sondern auch mit der damit verbundenen vom Publikum empfundenen Wahrnehmung von Freude und Erleichterung. Diese Funktion wird oft durch die nicht-ernsthafte Kommunikation bzw. Scherzkommunikation (cf. Kotthoff 1996) erfüllt, bei der die Ironie im Vordergrund steht.

In einem kommunikativen Kontext wie dem von Talkshows dient Ironie nicht nur als Strategie zur Ko-Konstruktion der Interaktion zwischen den in die Kommunikation involvierten Gesprächspartner, sondern sie wirkt auch auf die Parteien, die nicht direkt an dem Gespräch teilnehmen, aber an der Sendung beteiligt sind und auf unterschiedliche Weise in die Kommunikation involviert sind, i. e. das Publikum im Studio und die Zuschauer zu Hause.

Mit einem gesprächsanalytischen Ansatz zielt die vorliegende Arbeit darauf ab, die kommunikativen Funktionen und die pragmatischen Effekte ironischer Äußerungen in dem halbstrukturierten Rahmen einer Talk-Show zu untersuchen. Ein besonderes Augenmerk wird auf den Umgang mit ironischen Äußerungen zwischen den Interaktanten gelegt und auf die Beziehungen zwischen ihnen.

Die vorliegende Arbeit ist wie folgt strukturiert: Der erste Teil definiert das Phänomen der Ironie sowohl aus rhetorischer als auch aus pragmatischer Perspektive, und zwar sowohl im Hinblick auf systematische Komplexität als auch ihre kommunikativen Auswirkungen im Kontext von Fernseh-Talkshows. Dann werden sowohl konstitutive (Ironiefaktoren) als auch zusätzliche (Ironiemarkers) Elemente der Ironie dargestellt, die dabei helfen, Ironie innerhalb des Korpus zu identifizieren. Dann werden die Hauptmerkmale des TV-Talkshow-Genres erläutert, zusammen mit dem Untersuchungskorpus und dem angewendeten methodologischen Ansatz. Im Folgenden werden drei Beispiele von ironischen Äußerungen analysiert, in denen eine unterschiedliche Beteiligung der Interaktanten zur Schaffung von Gruppenbildung zu beobachten ist. Die Arbeit schließt sich mit einigen Schlussbemerkungen ab.

\footnotetext{
1 Für einen Überblick auf die Geschichte der Ironie im Hinblick auf Philosophie, Rhetorik und Literatur cf. weiterführend Morreal (1987) und Colebrooks (2004).

${ }^{2}$ Für einen Überblick auf Ironie im Zusammenhang mit Linguistik, Kognitions- und Sozialwissenschaften cf. weiterführend Barbe (1995) und Colston/Gibbs (2007).

${ }^{3}$ Einige Studien untersuchen das Phänomen der Ironie innerhalb der TV-Talkshow nur am Rande und in Bezug auf andere Themen, z. B. (Un)Höflichkeit (cf. Culpeper 2005).
} 


\section{Ironie: Formen und kommunikative Funktionen}

Laut der umfangreichen Literatur über Ironie gehen ihre Wurzeln auf die griechische und lateinische Rhetorik ${ }^{4}$ zurück: Ironie ist eine rhetorische Strategie, ${ }^{5}$ mit der das Gegenteil von dem verstanden wird, was wirklich ausgesprochen wurde (cf. Mizzau 1984: 16). Im Laufe der Jahrhunderte wurde der Kern dieser Definition beibehalten und erweitert, z. B. von Knox (1973: 25) und Lapp (1992: 24), die ihre Merkmale in vier Punkten zusammenfassen:

1. Das Gegenteil vom dem sagen, was man meint;

2. Etwas anderes sagen, als man meint;

3. Tadeln durch falsches Lob, loben durch vorgeblichen Tadel;

4. Jede Art des Sichlustigmachens und Spottens.

Von der Definition her wird hier auf der einen Seite auf den eigentümlichen antiphrastischen Aspekt der Ironie (1) und auf der anderen Seite sowohl auf ihre Zielsetzungen (3) als auch Aspekte hingewiesen, die sich auf einen weniger greifbaren Bereich beziehen, i. e. die Absichten und Überzeugungen des Sprechers (2). Darüber hinaus wird Ironie als eine Technik klassifiziert, die darauf abzielt, Spaß auf Kosten anderer zu haben (4). Diese Vier-Punkte-Definition hat den Vorzug, sowohl sprachliche als auch nicht-sprachliche Aspekte zu vermischen, aber Ironie scheint ein viel komplexeres Phänomen zu sein. Die Aussage, dass Ironie das Gegenteil von dem ist, was wörtlich ausgesprochen wird, scheint weder ausreichend noch immer wahr zu sein, z. B. wenn A einem Freund, der etwas Dummes getan oder gesagt hat, sagt: „Du bist ein Genie!“. Damit meint A nicht das Gegenteil, i. e. „Du bist kein Genie“, sondern gemeint ist, dass B sich in Bezug auf den Kontext, in dem sie sich befinden, unbeholfen/unangemessen verhalten hat. Ironie scheint also unabhängig von der syntaktischen Struktur der Äußerung zu sein, denn in der Tat sind nicht nur Aussagesätze (bei denen das Gegenteil leicht auszudrücken ist), sondern auch Fragesätze geeignet, Ironie zu erzeugen, z. B. „Fahren Sie ans Meer?“, die einem Kollegen gestellt wird, der sehr salopp gekleidet ist und eine Rede vor einem großen Publikum halten muss. Dann müssen Schlussfolgerungen gezogen werden, um den lokutionären Akt („Fahren Sie ans Meer?“) dem Kontext anzupassen (unangemessene Freizeitkleidung in einer formellen Situation) und sie mit dem enzyklopädischen Weltwissen zu vergleichen (keine Freizeitkleidung in einer formellen Situation tragen/eine formelle Kleiderordnung ist in formellen Situationen angebracht), um Zugang zu dem zu erhalten, was der Redner wirklich meinte (Er sollte sich besser anders kleiden).

Auf der kommunikativen Ebene ist Ironie immer riskant: der Zuhörer könnte beispielweise nicht wahrnehmen, was „,zwischen den Zeilen“ gesagt wird bzw. einen aus einem ironischen Ausdruck entstandenen Gesichtsbedrohenden Akt nicht akzeptieren, da Ironie meist dazu gedacht ist, eine negative Bewertung auszudrücken (cf. Kreuz/Link 2002: 128). Dank ihrer Indirektheit ist Ironie aber auch als gesichtswahrende Strategie gemeint; einerseits mildern ihre

\footnotetext{
${ }^{4}$ Für einen kurzen Überblick dieser Stilfigur im Bereich der Rhetorik cf. Mortara Garavelli (2018: 240f.).

${ }^{5}$ Das Wort Ironie stammt aus dem Griechischen eirōneía ,vortäuschen', das meist mit dem Ziel verwendet wird, den Diskurs zu verschönern und den Gesprächspartner zu überzeugen. Bereits Quintilian schreibt im 1. Jahrhundert v. Chr. in seiner Institutio Oratoria (in der Übersetzung von Rahn 1975: 288), dass ,,[...] Ironie als Figur von der Ironie als Tropus der Ganzen Gattung nach fast gar nicht verschieden - bei beiden nämlich handelt es sich darum, das Gegenteil vom dem zu verstehen, was ausgesprochen wird -, daß aber ihre Erscheinungsform verschieden sind, ist bei behutsamerem Hinsehen leicht festzustellen“.
} 
semantische Inversion und Indirektheit einen möglichen gesichtsbedrohenden Akt (cf. Brown/Levinson 1987), andererseits, wenn ein gesichtsbedrohender Akt die Kommunikation gefährdet, hat der Ironiker die Möglichkeit, sich zurückzuziehen und zu sagen, dass es nicht ernst gemeint war (Attardo 2000: 13).

Es ist außerdem immer noch unklar, warum ein Sprecher beschließt, eine Botschaft mit Ironie auszudrücken, die weniger verständlich ist und dem Zuhörer einen größeren kognitiven Aufwand abverlangt, um sie zu entziffern, anstatt sie wörtlich auszusprechen (Dews/Kaplan/Winner 2007).

Der, der Ironie verwendet, zeigt auf der einen Seite die Fähigkeit, sowohl sprachliche als auch sozio-pragmatische Merkmale der Sprache zu beherrschen (cf. Bettoni 2001:6) und seine Emotionen zu kontrollieren (Dews/Winner 1995: 347). Auf der anderen Seite erfordert die Ironie auch die Fähigkeit des Hörers, die Botschaft zu verarbeiten, um den wahren Inhalt der Botschaft zu verstehen.

Kurz: Ironie ist ein performatives Phänomen (cf. Nuoljiärvi/Tiittula 2011: 572), das viel mehr als eine semantische Inversion beinhaltet. Es handelt sich um eine komplexe Einstellung (cf. Bertuccelli 2018), um die Unzulänglichkeit einer bestimmten Handlung $\alpha$ in einem bestimmten Kontext $\beta$ hervorzuheben. Solche Unzulänglichkeit wird durch ein breites Spektrum sprachlicher (z. B. Übertreibung, Untertreibung etc., cf. Dynel 2016; Calpestrati 2020a) und paralinguistischer Mittel (Gestik, Mimik etc.) sowie komplexe kognitive Prozesse (Illokutionen, Erkennen des Gefühls und der geistigen Einstellung des Gesprächspartners etc.) vermittelt, die uns mehr Informationen über die Sprecher/Hörer-Beziehung geben als erwartet und die die interaktive Entwicklung der Kommunikation beeinflussen.

Für die Zwecke dieser Arbeit scheint es sinnvoll, zwischen den Faktoren zu unterscheiden, die die Ironie selbst konstituieren (Ironiefaktoren), und denen, die dem Hörer helfen und ihn dabei unterstützen, sie zu entdecken und zu erkennen (Ironiemarker).

\subsection{Ironiefaktoren}

Ironiefaktoren stellen sich als wesentliche Elemente der Ironie dar, ohne die sie nicht existieren könnte (cf. Attardo 2000; Burgers/van Mulken 2017). Zu den Ironiefaktoren zählt man Implizitheit, implizite und explizite Zielbewertung und semantische Inversion. ${ }^{6}$

Ironie muss zuerst implizit sein und Zuhörer bzw. Leser müssen das, was zwischen den Zeilen ausgedrückt wird, aus kontextuellen Hinweisen ableiten; wenn alle Inhalte wörtlich ausgedrückt würden, gäbe es in der Tat überhaupt keinen ironischen Effekt. Zweitens müssen ironische Äußerungen immer die Bewertung eines vorgegebenen Ziels beinhalten, i. e. das Ziel der Verhöhnung (cf. u. a. Kotthoff 2003; Bosco/Bucciarelli 2008). Die Bewertung kann offen oder verdeckt sein: Im ersten Fall wird sie explizit durch bestimmte lexikalische Verfahren angekündigt, indem die Semantik mit der Realität kollidiert, z. B. der Satz „Was für ein wunderbares Wetter heute", der an einem regnerischen und nebligen Novembertag geäußert wird. Hier steht diese Äußerung eindeutig im Widerspruch zu den tatsächlichen klimatischen Bedingungen. Der

\footnotetext{
${ }^{6}$ Für eine detaillierte Beschreibung von Ironiefaktoren mit Beispielen und weiterführende Literatur cf. Burgers/van Mulken/Schellens (2012) und Burgers/van Mulken (2017).
} 
Zuhörer kann jedoch auch aufgefordert werden, eine implizite (verdeckte) Bewertung abzuleiten, da es an expliziten Signalen fehlt, die an die Semantik der Äußerung gebunden sind und mit der tatsächlichen Realität kollidieren z. B. wenn ein Tourist an einem regnerischen Tag „Oh Toskana im Mai!“ ausruft (cf. Wilson/Sperber 1992: 55). ${ }^{7}$ In diesem Beispiel muss die Semantik der Äußerung mit kontextuellen Informationen abgeglichen werden: Wenn sie an einem sonnigen Frühlingsmorgen geäußert wird, muss der ganze Satz als Ausdruck von Freude verstanden werden, wenn sie dagegen an einem verregneten Frühlingsmorgen geäußert wird, müssen Schlussfolgerungen gezogen werden, um Zugang zur wahren Bedeutung des Satzes zu erhalten, weit entfernt von einem verträumten und leidenschaftlichen Ausdruck von Liebe zur Toskana. Drittens muss eine ironische Äußerung eine semantische Inversion enthalten, i. e. die wörtliche Bedeutung erzeugt Inkongruenzen ${ }^{8}$ entweder im Hauptkontext oder im Kotext, ${ }^{9}$ die der Zuhörer lösen muss, um die Absicht des Sprechers zu erfassen. Inkongruenzen können durch eine positive wörtliche Bedeutung entwickelt werden, um etwas Schlechtes oder Negatives zu bezeichnen (ironisches Lob), wie in dem Satz „,cool, du hast einen tollen Job gemacht“, der zu jemandem gesagt wird, der den Braten anbrennen ließ. Andernfalls können sie durch eine negative wörtliche Bedeutung entwickelt werden, mit der Idee, etwas zu loben (ironischer Tadel), wie in „Du bist ja ein Looser“, das zu einem Freund gesagt wird, der viel Zeit damit verbracht hat, sich über seine mangelhaften Kenntnisse zu beklagen, dann aber bei der Klassenarbeit eine Eins erhält.

\subsection{Ironiemarker}

Ironiefaktoren bilden das Grundgerüst des Phänomens, während Marker Elemente sind, die das Vorhandensein eines ironischen Inhalts signalisieren und die entfernt werden können, ohne die Ironie selbst oder zumindest ihre Erkennbarkeit zu beeinträchtigen (cf. Attardo 2000: 6). Solche Marker können auch unter dem Etikett der contextualisation cues aufgenommen werden, da sie zu dem Gespräch gehören und die Kontextualisierung unterstützen, i. e. sie machen die Interpretation einer bestimmten Äußerung relevant (cf. Auer 1992: 25). Contextualisation cues beziehen sich auf Signale verschiedener Art (morpho-syntaktische, paralinguistische etc.), die den Teilnehmern suggerieren, wie eine bestimmte Äußerung in einem bestimmten Kontext interpretiert werden muss (cf. Gumperz 1982: 131 f.; Auer/Di Luzio 1992: 24).

\footnotetext{
${ }^{7}$ Auch contextualisation clues (cf. 2.2) spielen eine Rolle und müssen beim ironischen Sprechen berücksichtigt werden, z. B. das Hintergrundwissen des Gesprächspartners, das enzyklopädisches Wissen, die sozialen Normen etc. (cf. Yus 1998). Ausgehend von der allgemeinen Annahme, dass Menschen keine regnerischen Tagen während der Ferien mögen, geht der Sprecher hier davon aus, dass das neblige Novemberwetter vom Gesprächspartner nicht als „wunderbar“ empfunden wird. Diese Annahmen müssen immer bestätigt werden, da es Menschen geben kann, die Nebel und Regen lieben, auch im Urlaub.

${ }^{8}$ Eine semantische Inversion besteht aus einer Inkongruenz zweier gegensätzlicher Skripte (cf. Attardo/Raskin 1991). Raskin gemäß (1985: 46) gemeint wird hier mit dem Begriff script "a large chunk of semantic information surrounding the word and evoked by it [...] a cognitive structure internalized by the native speaker".

${ }^{9}$ Wie in Burgers/van Mulken/Schellens (2012: 293) tritt eine Inkongruenz mit dem Kontenxt auf, wenn die wörtliche Bedeutung der Information mit der früheren gegebenen Information nicht übereinstimmt. Andernfalls wird eine Inkongruenz mit dem Kotext erzeugt, wenn die gegebene Information nicht übereinstimmt mit anderen Elementen, die in dem vorherigen Textausschnitt schon erwähnt wurden.
} 
Dabei handelt es sich um eine weit gefasste Kategorie, mit der sich viele Wissenschaftler auseinandergesetzt haben (cf. Mücke 1978; Kreuz 1996; Seto 1998; Attardo 2000; Burgers/van Mulken/Schellens 2012), die unter ihnen stilistische und morphologische Marker (cf. Kreuz 1996; Seto 1998; Burgers/van Mulken/Schellens 2012), prosodische Einheiten (cf. Cutler 1976; Kreuz/Robert 1995; Cheang/Pell 2009) oder visuelle und kinästhetische Bewegungen (cf. Rockwell 2001; Caucci/Kreuz 2012; Tabacaru/Lemmens 2014) identifiziert haben. Ironiemarker lassen sich auf verschiedenen Analyseebenen finden und untersuchen: Satz-Marker umfassen z. B. eine spezielle Interpunktion, die Schriftart bzw. das Layout; morpho-syntaktische Marker umfassen z. B. Negations- oder Topikalisierungstechniken. Kotextuelle und kontextuelle Marker umfassen sowohl linguistische Elemente wie die Verwendung von Metaphern oder anderen Tropen, als auch paralinguistische und visuelle Elemente. Nonverbale Elemente können außerdem eine wertvolle Hilfe bei der Erkennung von Ironie in gesprochenen Dialogen sein, in denen auch prosodische und visuelle Elemente vorhanden sind. Ironiefaktoren spielen eine grundlegende Rolle innerhalb einer multimodalen Analyse der Ironie, da sie nicht nur berücksichtigen, wie die Kommunikation vom Sprecher gestaltet wird, sondern auch, wie kontextuelle Hinweise und paralinguistische Praktiken die Kommunikation beeinflussen und zur Artikulation eines ironischen Inhaltes führen.

\section{$3 \quad$ Ironie in den Talkshows}

Unter Talkshow versteht man eine Fernseh- oder Radiosendung, in der eine Person oder eine Gruppe von Personen verschiedene Themen diskutiert und die von mindestens einem Gastgeber moderiert wird. Die Gäste sind in der Regel entweder gewöhnliche Personen oder Prominente, die Erfahrung mit dem Diskussionsthema der Sendung haben. Gäste werden häufig eingeladen, um ihre Geschichte mitzuteilen, zu diskutieren oder Fragen im Zusammenhang mit dem Thema zu beantworten. Solche Geschichten werden durch die Interaktion von Gästen und Gastgebern begleitet und sind meist ironisch und witzig (cf. Van Eimeren/Gerhard 1998; Krüger 1998; Gleich 1998 und 2001; Plake 1999; Schneiderbauer 2001; Girnth/Michel 2007; Keller 2009). In Bezug auf Prominenten-Interviews innerhalb von Talkshows (cf. Norrik, 2009) gibt der Moderator in der Regel mit einem Kommentar oder einer Frage einen Impuls zur Diskussion, und wenn die Gäste besonders gesprächig sind, unterbricht der Gastgeber ihre Rede nur selten.

Diese Art von Fernseh-Talkshow-Gesprächen werden als halbspontan bezeichnet, weil sie von den Moderatoren durch ein halb-strukturiertes bzw. Leitfadeninterview (cf. Edwards/Holland 2013: 2; Loosen 2016) initiiert werden, bei dem der Moderator das Gespräch durch vorab konstruierte Fragen leitet, die auf die Erörterung bestimmter Themen abzielen; allerdings besteht ein Spielraum für Unvorhersehbarkeit, da die Gäste frei antworten bzw. kommentieren können etc. Die Kommunikation in Talkshows scheint oft sehr dicht und informativ zu sein und manchmal ist es schwierig, eine Argumentationslinie zu erkennen, weil „die Argumentation in einer willkürlichen, oft chaotischen Weise abläuft (cf. einige sind repetitiv, einige nicht ganz auf den Punkt gebracht, einige unterbrochen, einige aneinander vorbei)“ (Haarman 1997: 79) und weil alle Interaktanten (Gäste, Publikum im Studio und zu Hause) unterschiedlich in die Kommunikation eingebunden sind.

Talkshows stellen sich deshalb als ,innermediale nicht-fiktionale Realität“ dar (cf. Burger/Lüginbuhl 2014: 209), i. e. eine solche rekonstruierte Realität existiert außerhalb des Studios nicht. 
Alle Beteiligten bekennen sich zu ihrer Rolle nur in diesem temporären Kontext, der nur dank und durch das Medium selbst möglich ist.

Auch wenn Talkshows in der Regel so konzipiert sind, dass sie den Zuschauern den Eindruck von Spontaneität vermitteln und suggerieren, dass die Kommunikation nur die Gäste vor der Kamera betrifft, umfassen sie viel mehr Aspekte als die, man nur am Bildschirm wahrnimmt. Die Kommunikation zwischen verschiedenen Rezipienten wurde als eine Reihe von imaginären Kreisen (Kommunikationskreise) beschrieben (cf. Burger/Lüginbuhl 2014: 25). Nach diesem Modell wird der innere Kreis in einer Fernseh-Talkshow von den Gästen und Moderatoren repräsentiert und deren Interaktion wird als multidirektional bezeichnet und ist doppeladressiert, da der im innersten Kreisen vermittelte Informationsfluss sich der sowohl an das Publikum im Studio (mittlerer Kreis) als auch an das Publikum vor dem Bildschirm (äußersten Kreis) richtet (cf. Burger/Lüginbuhl 2014: 25). Das Publikum, das im Studio mit Moderatoren und Gästen physisch anwesend ist, kann ebenfalls die Kommunikation beeinflussen, indem es entweder direkt oder durch para-soziale Interaktionen an der Diskussion teilnimmt (cf. van Eimeren/Gerhard 1998: 601). Die Rolle des Publikums kann innerhalb der Talkshows variieren, z. B. es kann das Gespräch unterbrechen und Fragen können gestellt werden, bzw. kann es nur Teil der Kulisse sein und somit schweigen, oder eventuell lachen oder applaudieren. Unabhängig von seinem Verhalten wird das Publikum in irgendeiner Weise von den Zuschauern zu Hause wahrgenommen, so dass das Publikum zu Hause auch Empfänger einer indirekten Kommunikation aus dem Mittelkreis wird.

Ein vorherrschender interpersonaler Diskurs entfernt das Gespräch von einem informativen Inhalt strictu sensu und bringt es einem klatschhaften Stil näher (cf. Burger/Luginbühl 2014: 336). Ironie ist nie unbeabsichtigt, und einige ihrer Auswirkungen auf die Kommunikation wurden bereits im Rahmen von Fernsehsendungen wie politischen Fernsehdebatten analysiert. Ironie wird vor allem zu einem Zweck eingesetzt: Distanz zur Zielscheibe der Ironie herzustellen (cf. Mizzau 1984: 95). Innerhalb der politischen TV-Talkshows offenbart der Einsatz ironischer Äußerungen eine kritische Haltung, die jede humoristische Absicht ausschließt (Kotthoff 2003: 1408) und die entweder als Verteidigung oder als Angriff gedacht ist (cf. Nuolijärvi/Tiittula 2011: 584). ${ }^{10}$

Bei diesem Anlass zeigt sich Ironie als ideales Mittel zur Analyse einer inneren medialen fiktiven Realität und der Doppeladressiertheit innerhalb eines TV-Talkshow-Gesprächs mit einer unterhaltenden Funktion. Obwohl jeder genau weiß, dass die Sendung ausgestrahlt wird und öffentlich auf dem Bildschirm zu sehen ist, tun die Gäste manchmal so, als ob der Informationsfluss innerhalb des innersten Kreises bleiben würde, was einen komischen Effekt auf das Publikum hat: ${ }^{11}$

\footnotetext{
10 Zur Anwendung ironischer Äußerungen als Angriff in der politischen Propaganda cf. u. a. Prestin 2000; Nuolijärvi/Tiittula 2011; Calpestrati/Foschi Albert 2019.

${ }^{11}$ Dieses Beispiel stammt aus dem Korpus der NDR-TV-Talkshow-Transkriptionen, die für diese Arbeit gesammelt wurden.
} 
(Beispiel 1)

Episode 17.11.2017, Min. 59:25

Jan Joseph Liefers (J); Jürgen Vogel (V); Barbara Schöneberger (B)

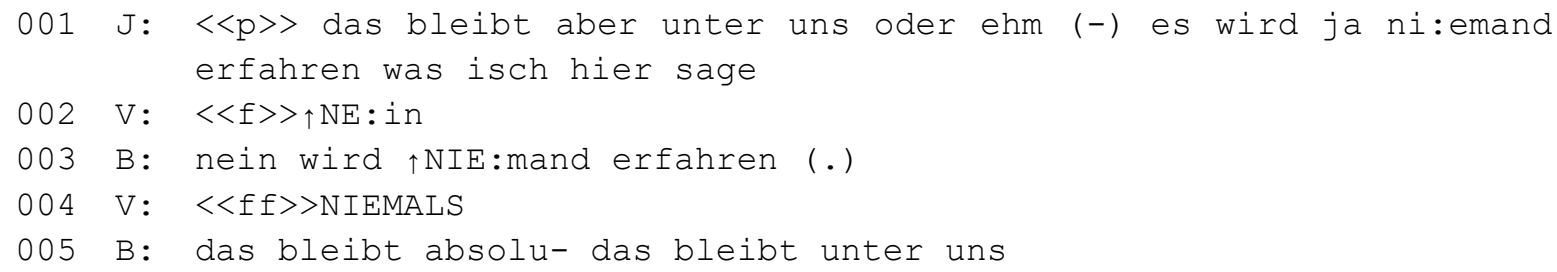

Jan Joseph Liefers, ein deutscher Schauspieler, will vorgeblich sicher sein, dass das, was er sagt, von niemandem gehört wird. Alle Antworten anderer Gäste und der Moderatoren wie „nein wird niemand erfahren“, oder „niemals“ werden ebenfalls mit einem ironischen Tonfall ausgesprochen, deren Markierungen (siehe 2.2) durch Vokaldehnungen und betonte Akzente explizit gemacht wird. Ironie wird hier von allen Teilnehmern verwendet, um indirekt zu zeigen, dass sowohl Gäste als auch Moderatoren sich der vermittelten fiktionalen Realität, in die sie vorübergehend eingebettet sind, bewusst sind.

\section{$4 \quad$ Methodologie und Korpusbeschreibung}

Der für die Analyse erstellte Korpus besteht aus 5 Sendungen der NDR-Talkshow, die zwischen August 2017 und November 2017 ausgestrahlt wurden. Diese Talkshow wird vom Norddeutschen Rundfunk produziert und dreimal im Monat, freitags, mit einer Dauer von ca. 120 Minuten pro Folge ausgestrahlt. Die NDR-Talkshow gehört zu den Late-Night-Chats (cf. Plake 1999: 87), bei denen zwischen acht und neun Gäste, sowohl Prominente als auch Experten eines bestimmten Gebietes, auf Sofas oder Sesseln um einen großen Tisch sitzen. Alle Gäste werden von den beiden derzeitigen Moderatoren, Barbara Schöneberger und Hubertus Meyer-Burkhardt, begrüßt, vorgestellt und dann interviewt. Gäste und Moderatoren befinden sich in der Mitte der Bühne, während die zur Show eingeladenen Zuschauer um sie herum sitzen; sie dürfen applaudieren und lachen, aber sie ergreifen nie das Wort und dürfen die Diskussion nicht unterbrechen. Das Studio hat eine konzentrische Form: Moderatoren und Gäste sitzen kreisförmig in der Mitte des Studios, die Zuschauer sitzen an kleineren Tischen, die außerhalb der „Runde“ angeordnet sind. Sowohl die Szenografie als auch die Möblierung schaffen eine selbstbewusste und gemütliche Atmosphäre, die dazu beiträgt, einen Eindruck von Intimität zu vermitteln und humorvolle Situationen leicht zu evozieren (cf. Jin/Wang 2012).

Ironie wird zunächst anhand des hörbaren Lachens identifiziert, das entweder von den Interagierenden oder vom Publikum kommt. Das Lachen hilft dabei, diejenigen Passagen im Text zu identifizieren, in denen Ironie zu finden ist. Es ist hier als bevorzugter (wenn auch nicht eindeutiger) ${ }^{12}$ Marker gedacht, um das Vorhandensein eines humorvollen Inhalts zu signalisieren (cf. Norrick 1993; Kotthoff 1999; Hay 2001; Calpestrati 2019), der durch eine ironische Äußerung kodifiziert werden kann (cf. Dynel 2009 und 2016; Burgers/van Mulken 2017: 394). Da Lachen auch unabhängig von einer komischen ironischen Äußerung hervorgerufen werden kann, wird hier nur das Lachen berücksichtigt, das auf den vorangegangenen humoristischen

\footnotetext{
12 Zur Funktion des Lachens als Marker von sowohl humoristischen als auch von nicht-humoristischen Inhalte cf. weiterführend Hay (2001), Provine (2001), Glenn (2010), Calpestrati (2020b).
} 
Kotext zurückzuführen ist. In Anlehnung an die oben gegebene Beschreibung von Ironie wird geprüft, ob die Textpassage eine rein beschreibende Funktion erfüllt oder ob sie eine Bewertung im Hinblick auf ein bestimmtes Ziel ist (cf. Burgers/van Mulken/Schellens 2012: 293). Schließlich werden die kontextuellen Faktoren analysiert, die die ironische Äußerung begleiten (wie z. B. eine besondere Prosodie, Gestik oder Körpersprache), um sowohl die Absicht des Sprechers als auch die Reaktionen des Hörers auf ironische Passagen und demzufolge die Gruppendynamik zur Schaffung konversationeller Zusammengehörigkeit zu erkennen.

\section{$5 \quad$ Ergebnisse der Untersuchung}

Die Präsenz von Ironie ist innerhalb der analysierten halbstrukturierten TV-Talkshow-Gespräche konsistent. In einem zehnstündigen TV-Talkshow-Korpus wurden 25 Belege gefunden im Vergleich zu 24 ironischen Sequenzen, die in einer zwanzigstündigen TV-Debatte von Kotthoff (2003) gefunden wurden. Ironie gilt als rhetorische Technik, die sich als eine Art Verführungskunst darstellt. Sie wirkt nämlich unterschiedlich auf die kommunikativen Kreise, um eine unterhaltsame Hauptfunktion zur Schaffung von Gruppenbildung zwischen den verschiedenen Gesprächspartner zu erzeugen. Ironie kann eine Verbindung schaffen zwischen dem innersten und dem mittleren Kreis (Gäste und Publikum) (5.1) bzw. zwischen spezifischen Sprechern im innersten Kreis (Gäste und Moderatoren) (5.2), oder mit all denen, die in die Kommunikation involviert sind (5.3).

\subsection{Verbindung zwischen dem innersten und mittleren Kreis (Gäste und Publikum)}

Auch wenn Ironie vor allem wegen ihrer negativen Bewertung als potentiell aggressive verbale Handlung angesehen werden kann, gilt sie als verbale Technik, deren spöttische Wirkung nur dann gelingt, wenn der Ironiker auf eine solide Beziehung zum Gesprächspartner setzt (cf. Hartung 1996) oder wenn der Ironiker sich im Gespräch bewusst so positioniert (cf. Harré/Davies 1990), dass das Publikum die humoristische Absicht versteht. In (2) wirkt sich die spöttische Absicht des Sprechers auf den eher inneren und auf den äußeren Kommunikationskreis aus, die darauf mit Lachen reagieren, was gleichzeitig sowohl eine Verbindung mit dem Sprecher als auch Distanz der Zielperson gegenüber schafft.

Barbara, die Moderatorin, interviewt Elyas M'Barek, einen deutschen Schauspieler, der eingeladen wurde, über seinen neuesten Film zu sprechen. Barbara fragt Elyas, wie er seine letzte Woche verbracht hat. Er sagt, er sei in New York gewesen. Nach einer Weile unterbricht sich die Moderatorin und fragt Elyas mit verwunderter Stimme, warum er nicht zu Hause geblieben sei, um den Start seines neuen Films vorzubereiten, anstatt in Urlaub zu fahren.

(Beispiel 2)

Episode 20.10.2017, Min. 2:51

Barbara Schöneberger (B); Elyas M’Barek (E); Johann Lafer (J); Hubertus Meyer-Burkhart (H)

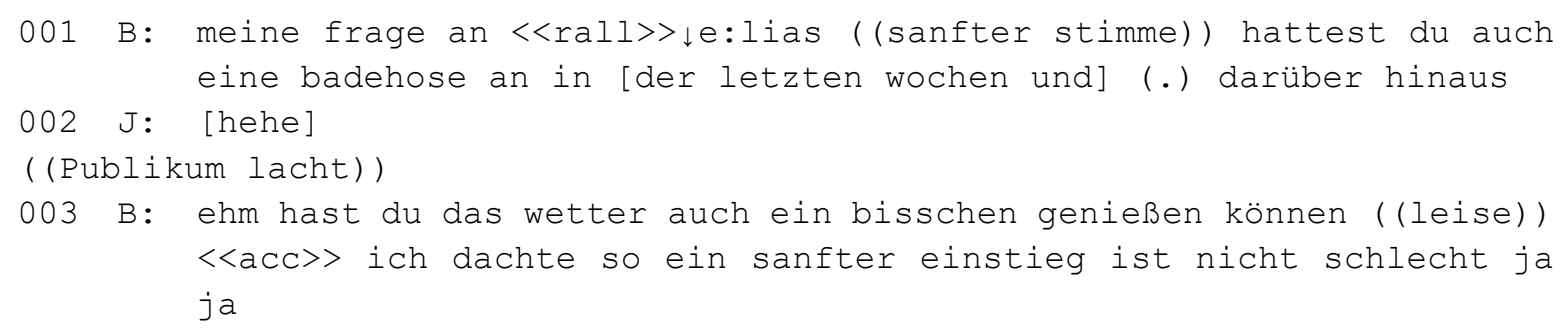




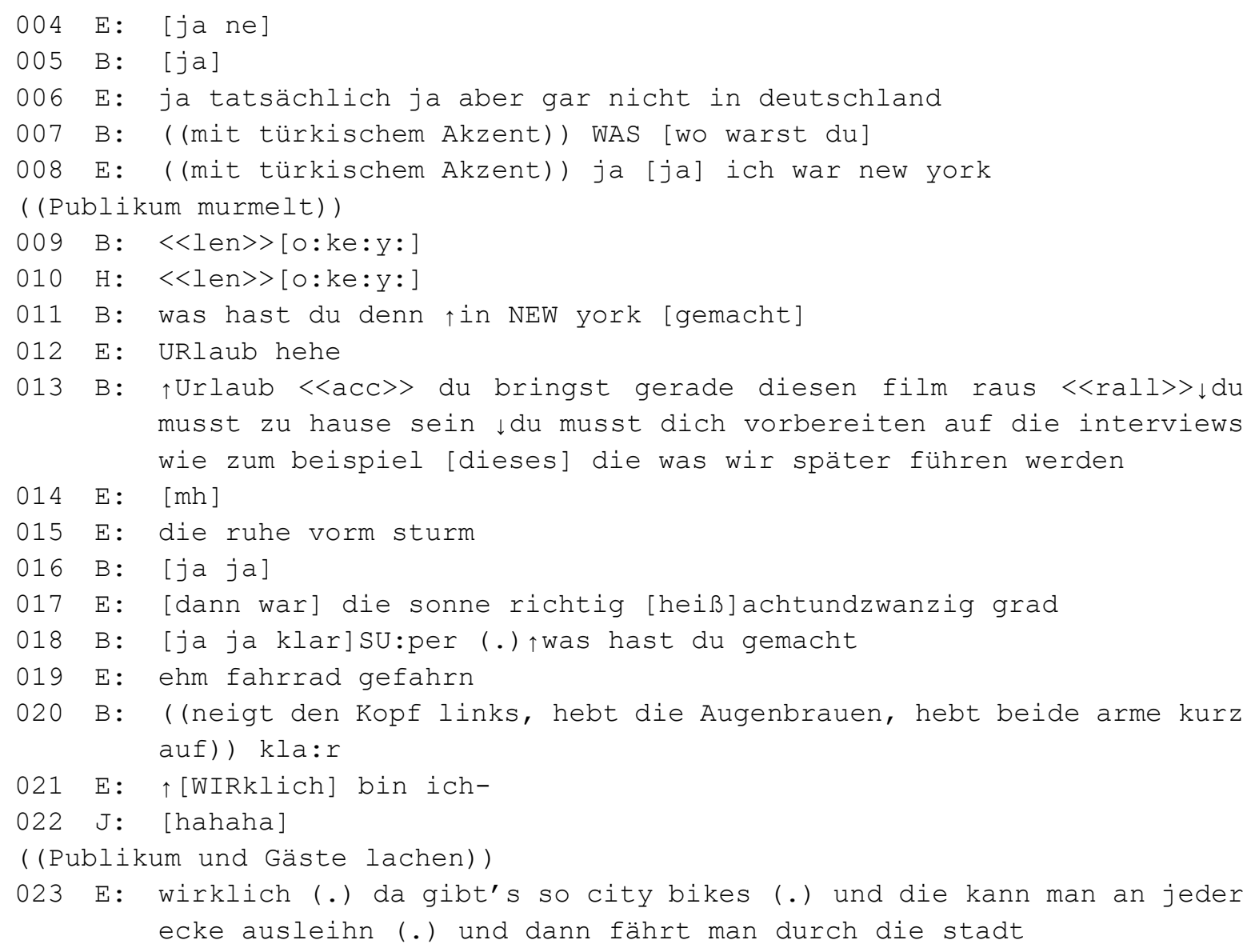

Von Anfang an lässt die Moderatorin das Publikum ihre scherzhafte Haltung gegenüber dem Befragten verstehen, indem sie ihren Tonfall (001) hörbar verändert, was das Gelächter des Publikums hervorruft. Dann senkt sie plötzlich ihren Tonfall, indem sie Elyas (003) zuflüstert und dabei eine intime Umgebung vortäuscht. Nach dieser Wendung fährt sie mit einem klaren und gut hörbaren Tonfall fort. Dann geht das humorvolle Gespräch auf Kosten des Befragten weiter, als Barbara, immer mit der Stimme spielend, den sogenannten Kiezdeutschen (007) parodiert, um sich über Elyas' türkische Wurzeln lustig zu machen. Barbaras Absicht, Elyas in eine benachteiligte Position zu versetzen (cf. Dews/Winner 1995: 348), kommt nicht wirklich zustande, da seine Antwort zeigt, dass er den Spott verstanden hat (008) (cf. Lee/Tanaka 2016: 2) und mit einem Satz reagiert, der die Morpho-Syntax des Kiezdeutschen widerspiegelt, in dem manchmal Präpositionen fehlen (cf. Wiese 2012: 59). Das humorvolle Moment wird dann noch durch ein Einverständnis zwischen den Gastgebern verstärkt, nämlich dass sie sich schnell anschauen und mit einer Silbenverlängerung „okay“ (009-010) ausrufen, was dazu beiträgt, die Idee zu vermitteln, dass Elyas etwas sehr Schickes getan hat. Als der Befragte verrät, dass er Fahrrad gefahren ist, hebt die Moderatorin die Arme leicht, beugt den Kopf nach links und ruft mit einem ironischen Tonfall ${ }^{13}$ „klar“ aus (020), der im Vergleich zu dem hektischen Tonfall der vorherigen Gesprächsbeiträge extrem still ist. Der propositionale Inhalt und die paralinguistischen und gestischen Hinweise (cf. Burgers/van Mulken 2017; Attardo et al. 2003; Rockwell 2001) kollidieren mit dem vorhergehenden Kotext und lassen uns erkennen, dass sie sich der

\footnotetext{
${ }^{13}$ Die Besonderheit des sogenannten ironischen Tons besteht in einem prosodischen Kontrast im Vergleich zum Tonfall, der für die vorherigen Gesprächsbeiträge verwendet wurde (cf. Bryant 2010).
} 
Ironie bedient, um ihren Gast zu verspotten. Diese ironische Wendung verwandelt sich einerseits in eine Verspottung auf Kosten des Befragten und andererseits in ein Zeichen der Zugehörigkeit und Gruppenbildung mit den Gästen und dem Publikum im Studio, die mit Lachen reagieren. Der Gast reagiert dann schnell mit einer Äußerung, aber er bleibt stehen und schaut sich dann um, als er versteht, dass er Opfer des Spotts der Moderatorin ist. Seine Antwort mit einem höheren Tonfall (021) verrät, dass er Barbaras ironische Wendung sofort versteht, tatsächlich reagiert er auf das implizit Gesagte. Als die anderen Gäste im Kreis anfangen zu lachen und alle Leute im Studio sich über den Spott von Barbara amüsieren, erklärt er erneut, er sei in New York wirklich Fahrrad gefahren. Seine Reaktion zeigt, dass der Zuhörer die kommunikativen Absichten des Sprechers nicht verfolgt, denn sie potentiell als Angriff auf das positive Gesicht empfunden werden kann (cf. Brown/Levinson 1987). Elyas Antwort bringt das Gespräch auf eine neutralere Kommunikationsebene zurück, die keine spielerische Dimension aufweist.

\subsection{Verbindung innerhalb des innersten Kreises (Gästen und Moderatoren)}

Ironie ist eine rhetorische Technik, die vor allem dazu dient, humorvolle Effekte zu erzeugen und das Publikum zu unterhalten, auch wenn sie manchmal das pragmatische Gesicht der Zielperson beschädigt. Wenn die Intentionen des Sprechers gut kodifiziert sind (z. B. die Absicht, Spaß zu haben oder die Leute zum Lachen zu bringen) und vom Zuhörer akzeptiert werden, fördert Ironie die kooperative Ko-Konstruktion (cf. Stivers/Mondada/Steensig 2011: 21) und demzufolge die Gruppenbildung. Im folgenden Beispiel bittet der Moderator Hubertus MeyerBurkhardt die deutsche Schauspielerin Veronica Ferres über einige lustige Episoden zu sprechen, die sich während der Dreharbeiten ihres neuesten Films ereignet haben. Der Gast fängt an, über einige witzige Ereignisse zu sprechen, bis die beiden Moderatoren beschließen, sich einzumischen und sich auf den Gast und das Gesprächsthema ironisch einzubringen.

(Beispiel 3)

Episode 22.09.2017, Min. 14:07

Hubertus Meyer-Burkhardt (H); Veronica Ferres (V); Barbara Schöneberger (B); unspecified guest $(\mathrm{G})$

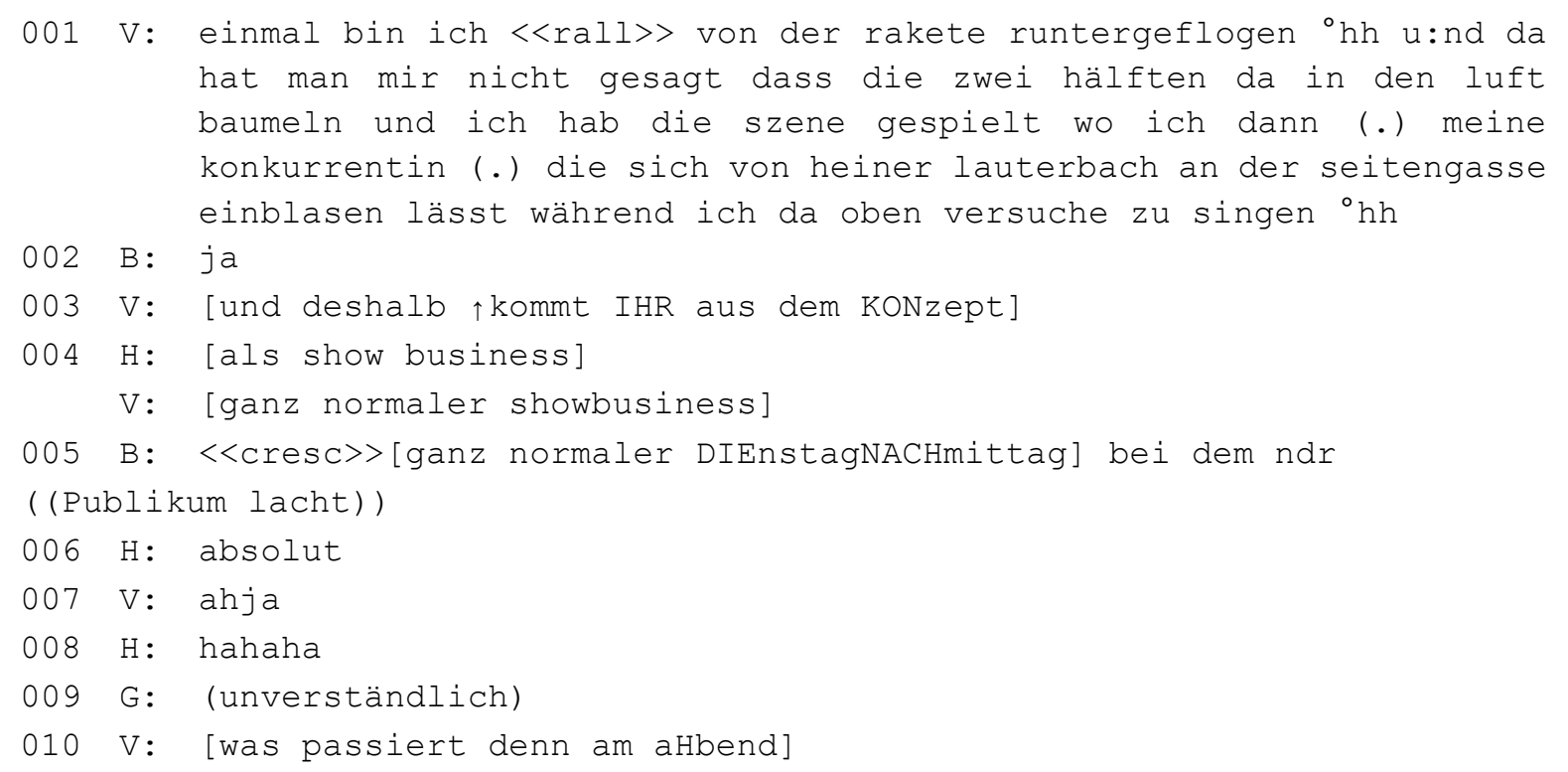




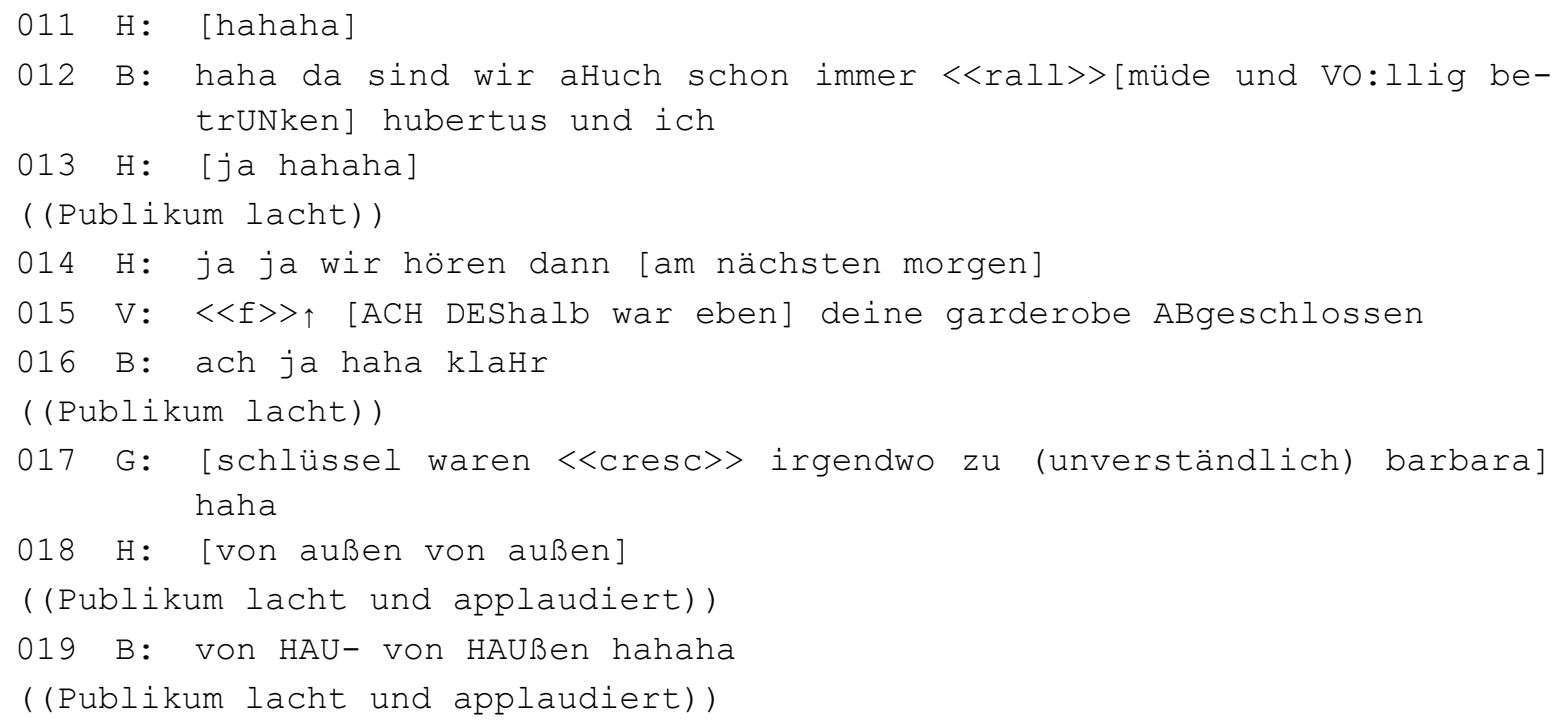

Während der Gast beginnt, einige lustige Geschichten zu erzählen, die am Set des Films passiert sind, kommentiert Hubertus Meyer-Burkhardt sarkastisch, dass das, was passiert ist, auf das übliche Showbusiness zurückzuführen ist. An dieser Stelle greift die zweite Moderatorin, Barbara Schöneberger, mit einem höheren Tonfall ein (005), der ihre ironische Haltung deutlich macht, fügt hinzu, dass all die merkwürdigen Dinge, die von dem Gast erzählt werden (als normale Aktivitäten dargestellt), früher an den Nachmittagen des NDR passierten. Hubertus stimmt mit Barbara überein (006) und lacht dann (008). Der Gast hört auf, seine Anekdote zu erzählen und beginnt mit einem neuen Gesprächsbeitrag (010) eine unerwartete humorvolle Dialogizität mit beiden Moderatoren. Auslöser dieser lustigen Szene ist die ironische Äußerung von Barbara (005), die die NDR-Talkshow als einen Ort bewertet, an dem merkwürdige Dinge passieren. Auf alle Gespräche folgt das Lachen der Gäste und des Publikums im Studio. Zusammen mit ihrem Gesprächspartner schaffen sie eine Art kleineren Kommunikationskreis, in dem alle drei zusammenarbeiten, um eine lustige Geschichte zu kreieren, die das Publikum unterhalten und zum Lachen bringen soll. Ironie dient hier als Technik, um eine humoristische Dimension zu schaffen: Alle involvierten Teilnehmer müssen Witz zeigen, um den Dialog interaktiv zu ko-konstruieren. Außerdem akzeptieren die beiden Moderatoren, mit dem Gast mitzuspielen, und die Rollen ändern sich für eine Weile: Die Moderatoren, Angelpunkt des Programms, werden plötzlich zur Zielscheibe des Humors, der durch den Gast ausgelöst wird (010 und 015). Die Harmonie und Synchronizität zwischen den Moderatoren ist sehr produktiv (cf. Burger/Luginbühl 2014: 326), da sie so etwas Ungeschriebenes wie humorvolle Geschichten produzieren können. Auch wenn Humor in manchen Fällen als gesichtsbedrohend empfunden wird, z. B. (005 oder 015) gefährdet er selten die Kommunikation und verstärkt im Gegenteil die Gruppenbildung (cf. Culpeper 1996: 353) und unterstützt gleichzeitig die Unterhaltungsfunktion der Sendung.

\subsection{Zwei Fliegen mit einer Klappe schlagen: Selbstironie}

Im Falle der Selbstironie nutzt der Sprecher die Ironie zur Selbsteinschätzung und verändert damit die kommunikativen Rollen: Der Sprecher ist nun selbst Ziel seines Spotts. Obwohl sie hauptsächlich dazu dient, eine negative Bewertung auszudrücken, offenbart sich Selbstironie als Strategie zur Selbstpositionierung (cf. Jones/Pittmann 1982) innerhalb der Talkshow. 
Barbara interviewt ihren Gast, Elyas M'Barek und sie sprechen über Essgewohnheiten und die Notwendigkeit, viel Flüssigkeit zu sich zu nehmen. Als der Befragte zugibt, dass er gewöhnlich viel Wasser trinkt, nutzt Barbara das, um einen humorvollen Moment zu schaffen.

(Beispiel 4)

Episode 20.10.2017, Min. 1:39:35

Barbara Schöneberger (B); Elyas M'Barek (E)

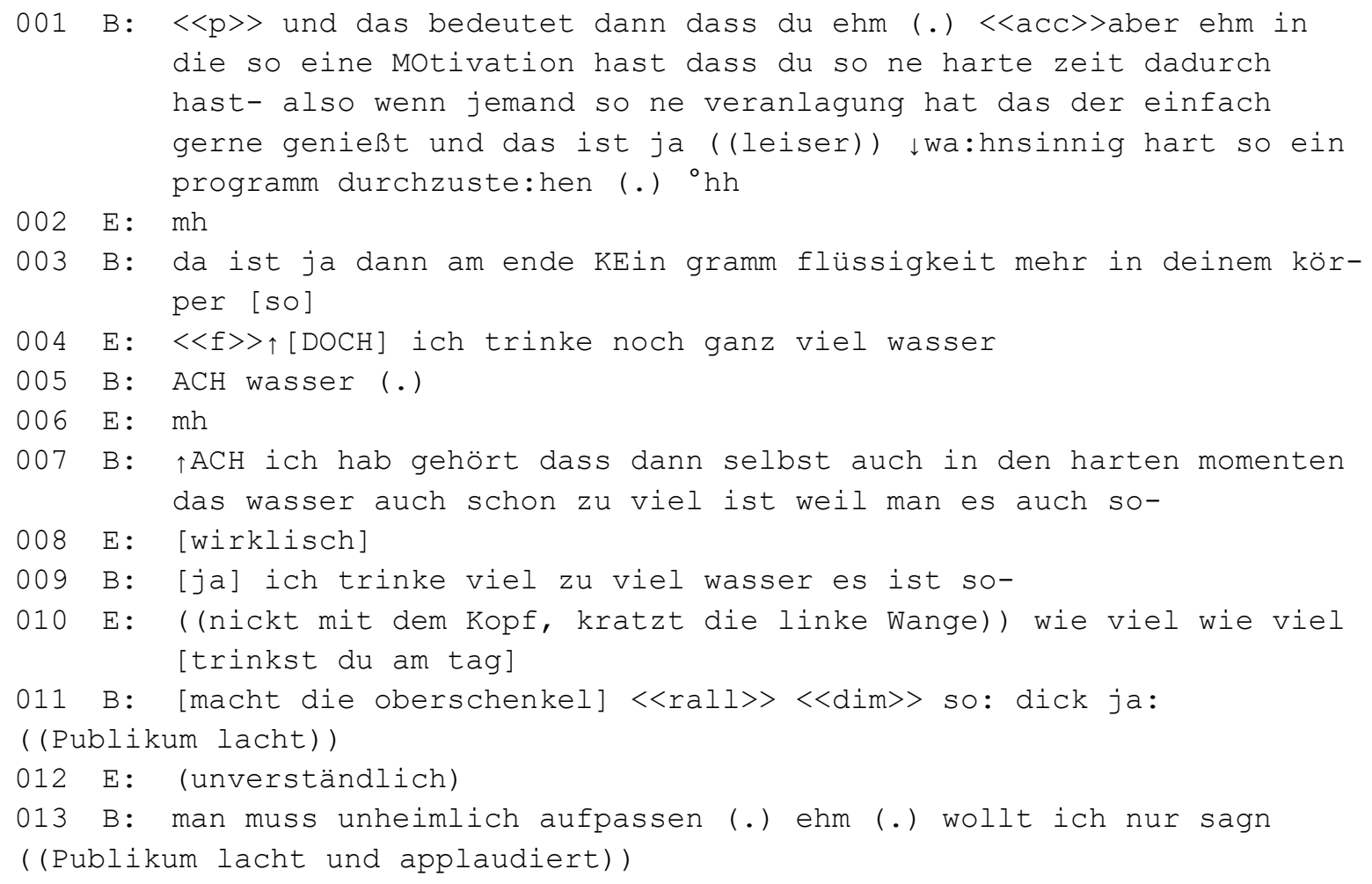

Bei (007) kommt Barbara an die Reihe und sagt, sie habe gehört, dass zu viel Wasser in den schwierigsten Momenten ungeeignet sein kann. Barbara senkt ihren Tonfall, wölbt die Augenbrauen und sagt dann mit ernstem Blick, dass zu viel Wasser die Oberschenkel dick macht. Während sie ihre Äußerung macht, nickt sie und kratzt sich an der linken Wange, tut so, als würde sie etwas Objektives sagen und sucht die Zustimmung anderer Gäste. Sowohl Gäste als auch das Publikum reagieren mit Gelächter. Mit demselben Ton fährt sie fort und liefert eine weitere ironische Wendung (013), die mehr Gelächter hervorruft und den humorvollen Moment beendet. Barbara hat einen Zusammenhalt zwischen den Interaktanten geschaffen, der im innersten Kreis anfängt und sich nach außen verbreitet. Diese ironische Äußerung (013) wird durch lexikalisch-grammatikalische Marker vermittelt, i. e. durch das verstärkende Adjektiv „unheimlich“, das ironisch verwendet wird, um den vorherigen Inhalt stark zu betonen und ihn als sehr wichtig zu bewerten, obwohl er es nicht ist. Barbara bewertet sich selbst implizit als fett und benutzt die Überhydratisierung als Entschuldigung. Die Semantik ihrer Äußerungen kollidiert mit dem allgemeinen Weltwissen, denn es ist kontra-intuitiv zu denken, dass Wasser zu Fett führt. Darüber hinaus vermitteln die Körpersprache und ihr flacher Tonfall ein ungewöhnliches Gefühl von Verlässlichkeit und Ernsthaftigkeit, das im Gegensatz zum vorherigen Gesprächssegment und zu ihrer heiteren und dynamischen Persönlichkeit steht. Barbara benutzt sich selbst als Zielscheibe ihrer Ironie und demonstriert damit ihre Absicht, sowohl die Gäste als auch das Publikum zu unterhalten; tatsächlich wirkt sich die ironische Wendung positiv auf 
sie aus, da die Zuschauer mit Lachen reagieren. Barbara zeigt sich auch nicht nur als kompetente Rednerin (cf. Bettoni 2001: 6), sondern auch als kluge und geistreiche Fernsehfrau, die in der Lage ist, humoristische Hinweise im Gespräch zu erfassen und in einen ironischen Satz umzuwandeln. In Anlehnung an Krieger (2002: 22) „der Moderator repräsentiert seine Sendung, er bildet eine untrennbare Einheit mit ihr", etabliert sich Barbara als Epizentrum der Szene, die alle Teilnehmer zum Lachen bringt, von denen im innersten bis zu denen im äußersten Kreis.

\section{$6 \quad$ Fazit und Ausblick}

In den halbstrukturierten Gesprächen zeigt sich Ironie als spontane kommunikative Strategie, die aus einem kreativen Intellekt heraus entsteht. Durch sprachliche wie auch nicht-sprachliche Elemente wird eine kognitiv herausfordernde ironische Äußerung formuliert. Darin erkennt man Ironie als rhetorische Technik, die in dem untersuchten Kontext meistens eine unterhaltsame Funktion hat: Aufgrund ihres geringen Aussagegehalts wirkt die Ironie meist auf die sozialen Beziehungen zwischen den Gästen im Kommunikationskreis.

Die Korpusanalyse hat gezeigt, dass Ironie Gruppenbildung zwischen den unterschiedlich aber gleichzeitig in dem Gespräch involvierten Kommunikationskreisen verursacht. Ironie schafft Verbindung zwischen den Interaktanten, die zu verschiedenen Kommunikationskreisen gehören bzw. zwischen Interaktanten, die sich in dem gleichen Kreis befinden. Auch wenn Ironie einer meist negativen Bewertung ausdruckt, führt sie zu keinem gesichtsbedrohenden Akt, denn die Unterhaltungsfunktion in dieser Talkshow vorwiegend ist (5.1). Im Gegenteil kann Ironie als Anreiz zur Ko-Konstruktion einer spaßigen Dimension führen (5.2) und sich an die zwei externen Kommunikationskreise richten, i. e. das Publikum im Studio und die Zuschauer zu Hause, für die das Programm konzipiert ist. (Selbst)Ironie kann außerdem Gruppenbildung zwischen allen Interaktanten schaffen, die zu den drei verschiedenen Kreisen gehören. Die Aufmerksamkeit aller Kommunikationskreise (Moderatoren, Gäste, Publikum und Zuschauer) richtet sich auf den Sprecher, worauf die potentielle negative Bewertung richtet (5.3): Barbara Schöneberger drängt sich in diesem Fall als eine Art Identifikationsobjekt auf (cf. Krieger 2002: 22), z. B. als geistreiche Moderatorin, die beim Interview oft Ironie einsetzt.

Im Allgemeinen zeigen die Korpusdaten, dass Ironie im Zusammenhang mit dem untersuchten Typus von Talkshow meist als verführerische Rhetorikstrategie gedacht ist, die hauptsächlich darauf abzielt, die Aufmerksamkeit des Zuhörers zu fesseln und eine freundliche und kooperative Dimension - wie in einer informellen Gesprächssituation unter Freunden - zu schaffen. Im Gegensatz zu den Ergebnissen sowohl von Nuolijärvi/Tiittula (2011) als auch von Kotthoff (2003) kann dies auf die Art der analysierten Talkshow zurückzuführen sein: In politischen Debatten intendiert Ironie meist einen Angriff auf den Gesprächspartner, während sie im Persönlichkeitsgespräch als unterhaltsame Strategie benutzt wird. Wenn ironische Äußerungen vom Zuhörer als gesichtsbedrohender Akt empfunden werden, dann sind die Reaktionen ähnlich wie die Ergebnisse von Nuolijärvi/Tiittula (2011): Der Wechsel zu einem nicht spöttischen Modus seitens des Zuhörers scheint die geeignetste Strategie zu sein, um einen Streit oder den Zusammenbruch der Kommunikation zu verhindern.

Für zukünftige Forschungen wäre es produktiv, den Korpus zu erweitern und zu beobachten, $\mathrm{ob}$ andere Interaktionsmodalitäten zwischen den Kommunikationskreisen zu finden sind und 
ob ironische Äußerungen immer mit einer verführenden Funktion gemacht werden. Aus diesem Grund wäre es interessant, verschiedene Talkshow-Genres zu vergleichen, um zu überprüfen, ob andere Kontexte die Funktionen desselben Phänomens beeinflussen können.

\section{Transkriptionskonventionen}

[]

(.)

$(-)$

:

ehm

haha hehe

wor-

gooHd

acCENT

${ }^{\circ} \mathrm{h}$

$\mathrm{h}^{\circ}$

$\uparrow$

$\downarrow$

$<<\mathrm{f}>>$

$<<\mathrm{p}>>$

$<<$ len $>>$

$<<$ cresc $>>$

$<<\operatorname{dim}>>$

$<<$ acc $>>$

$<<$ rall $>>$

((steht auf))

(unverständlich)
Überlappungen und Simultansprechen

kurze Pause

längere Pause zwischen 0.25 und $0.75 \mathrm{Ms}$.

Dehnung

Verzögerungssignale

Lachen

Abbruch

integriertes Lachen

Fokusakzent

Einatmen

Ausatmen

kleinere Tonhöhesprunge nach oben

kleinere Tonhöhesprunge nach unten

forte

piano

lento

crescendo

diminuendo

accelerando

rallentando

paraverbale Merkmale

unverständlich

\section{Literaturverzeichnis}

Attardo, Salvatore (2000): "Irony Markers and Functions: Towards a Goal-Oriented Theory of Irony and Its Processing". Rask 12: 3-20.

Attardo, Salvatore/Raskin, Victor (1991): "Script Theory Revis(it)ed: Joke Similarity and Joke Representation Model". Humor 4/3-4: 301-302.

Attardo, Salvatore et al. (2003): "Multimodal Markers of Irony and Sarcasm". Humor 16/2: 243-260.

Auer, Peter (1992): “Introduction: John Gumperz' Approach to Contextualisation”. In: Auer, Peter/Di Luzio, Aldo (eds.): The Contextualisation of Language, Amsterdam/Philadelphia: Benjamins.

Auer, Peter/Di Luzio, Giovanni (1992): The Contextualisation of Language. Amsterdam/Philadelphia: Benjamins.

Barbe, Katharina (1995): Irony in Context. Amsterdam/Philadelphia: Benjamins.

Bertuccelli, Marcella (2018): "Irony as a Complex Attitude" Lingue e Linguaggi 26: 59-80.

Bettoni, Camilla (2001): Imparare un'altra lingua. Roma/Bari: Laterza. 
Bosco, Francesca M./Bucciarelli, Monica (2008): "Simple and Complex Deceits and Ironies". Journal of Pragmatics 40/4: 583-607.

Brown, Penelope/Levinson, Stephen (1987): Politeness: Some Universals in Language Usage. Cambridge: Cambridge University Press.

Bryant, Gregory (2010): "Prosodic Contrasts in Ironic Speech". Discourse Processes 47/7: 545-566.

Burger, Harald (2005): Mediensprache. Eine Einführung in Sprache und Kommunikationsformen der Massenmedien. Mit Beitr. von Martin Luginbühl. Berlin/New York: De Gruyter.

Burger, Harald/Luginbühl, Martin (2014): Mediensprache. Eine Einführung in Sprache und Kommunikationsformen der Massenmedien. Berlin/Boston: De Gruyter.

Burgers, Christina/van Mulken, Margot (2017): "Humor Markers”. In: Attardo, Salvatore (ed.): The Routledge Handbook of Language and Humor. New York/London: Routledge: 385399.

Burgers, Christian/van Mulken, Margot/Schellens, Peter (2012): "Verbal Irony: Differences in Usage Across Written Genres”. Journal of Language and Social Psychology 31/3: 290-310.

Calpestrati, Nicolò (2019): "La comicità nel parlato spontaneo Tedesco: oggetti semantici e mezzi linguistici che producono la risata”. Studi Germanici 15/16: 189-206.

Calpestrati, Nicolò (2020a): „Sprachliche Mittel der Komik im gesprochenen Deutsch: Eine Projektskizze“. In: Auteri, Laura/Foschi Albert, Marina (eds.): Jahrbuch für internationale Germanistik. Jahrgang LII/Heft 1. Bern etc., Lang: 131-144.

Calpestrati, Nicolò (2020b): "La risata nella conversazione asimmetrica tedesca. Analisi di alcune funzioni socio-pragmatiche nell'interazione studente-docente". Rivista Italiana di Studi sull'Umorismo 3/1: 3-15.

Calpestrati, Nicolò/Foschi Albert, Marina (2019): "Ironie in der Propaganda der extremen Rechten". In: Schiewe, Jürgen/Niehr, Thomas/Moraldo, Sandro M. (eds): Sprach(kritik)kompetenz als Mittel demokratischer Willensbildung. Sprachliche In- und Exklusionsstrategien als gesellschaftiche Herausforderung. Bremen, Hempen: 151-164.

Caucci, Gina/Kreuz, Robert (2012): "Social and Paralinguistic Cues to Sarcasm". Humor 25/1: $1-22$.

Cheang, Henry/Pell, Marc (2009): "Acoustic Markers of Sarcasm in Cantonese and English". The Journal of the Acoustical Society of America 126/3: 1394-1405.

Colebrooks, Claire (2004): Irony. London/New York: Routledge.

Colston, Herbert/Gibbs, Raymond (2007): Irony in Language and Thought: A Cognitive Science Reader. New York: Erlbaum.

Culpeper, Jonathan (1996): "Towards an Anatomy of Impoliteness". Journal of Pragmatics. 25/3: 349-367.

Culpeper, Jonathan (2005): "Impoliteness and Entertainment in the Television Quiz Show: The Weakest Link". Journal of Politeness Research. Language, Behaviour, Culture 1/1: 35-72.

Cutler, Anne (1976): "Beyond Pausing and Lexical Look-Up: An Enriched Description of Auditory Sentence Comprehension". In: Wales, Rogers/Walker, Edward. (eds.): New Approaches to Language Mechanisms. Amsterdam, North Holland Linguistics Series: 133149.

Dews, Shelly/Winner, Ellen (1995): "Why Not Say It Directly? The Social Functions of Irony". Discourse Processes 19: 347-367. 
Dews, Shelly/Kaplan, Joan/Winner, Ellen (2007) "Why Not Say It Directly? The Social Functions of Irony". In: Gibbs, Raymond/Colston, Herbert (eds.): Irony in Language and Thought: A Cognitive Science Reader. New York: Erlbaum: 297-318.

Dynel, Marta (2009): "Beyond a Joke: Types of Conversational Humor". Language and Linguistic Compass 3/5: 1284-1299.

Dynel, Marta (2016): "Two Layers of Overt Untruthfulness. When Irony Meets Metaphor, Hyperbole or Meiosis". Pragmatics and Cognition 23/2: 259-283.

Edwards, Rosalind/Holland, Janet (2013): What is Qualitative Interviewing? London/New York: Bloomsbury.

Girnth, Heiko/Michel, Sascha (2007): „Von diskursiven Sprechhandlungen bis Studiodekorationen. Polit-Talkshows als multimodale Kommunikationsräume“. Der Sprachdienst 3: 8599.

Gleich, Ulli (1998): „Talkshows im Fernsehen - Inhalte und Wirkungen, Zuschauer- und Kandidatenmotive“. Media Perspektiven 12: 625-632.

Gleich, Ulli (2001): „Populäre Unterhaltungsformate im Fernsehen und ihre Bedeutung für die Zuschauer. Forschungsüberblick zu Nutzungsmotiven, Funktionen und Wirkungen von Soap Operas, Talkshows und Reality-TV“. Media Perspektiven 10: 524-532.

Gumperz, John (1982): Discourse Strategies. Cambridge: Cambridge University Press.

Haarman, Louann (1997): "Argument Style and Performance in the Audience Discussion Show”. In: Bondi, Marina/Bussi, Elisa/Gatta, Francesca. (eds.): Understanding Argument: La Logica Informale del Discorso. Bologna, CLUEB: 71-90.

Harré, Rom/Davies, Bronwyn (1990): "Positioning: The Discursive Production of Selves". Journal for the Theory of Social Behaviour 20: 40-63.

Hartung, Martin (1996) „Ironische Äußerungen in privater Scherzkommunikation“. In: Kotthoff, Helga (ed.) Scherzkommunikation. Beiträge aus der empirischen Gesprächsforschung. Oppladen, VS Verlag für Sozialwissenschaften: 109-143.

Hay, Jennifer (2001). The Pragmatics of Humor Support. Humor 14/1: 55-82.

Jin, Shengxi/Wang, Bin (2012) "A Relevance Theoretic-Based Approach to Verbal Humor in Joe Wong's Talk Xhow”. International Journal of English Linguistics 2/3: 44-48.

Jones, Edward/Pittmann, Thane (1982): "Toward a General Theory of Strategic Self-Presentation”. Psychological Perspectives of the Self 1/1: 231-262.

Keller, Harald (2009): Die Geschichte der Talkshow in Deutschland. Frankfurt a. M.: Fischer.

Knox, Norman (1973): „Die Bedeutung von ,Ironie“: Einfuhrung und Zusammenfassung“. In: Hass, Hans-Egon/Mohrlüder, Gustav-Adolf (eds.) Ironie als literarisches Phänomen. Köln, Kiepenheuer \& Witsch: 21-30.

Kotthoff, Helga (1996): Scherzkommunikation. Beiträge aus der empirischen Gesprächsforschung. Opladen: Springer Verlag.

Kotthoff, Helga (1999): “Coherent Keying in Conversational Humour: Contextualising Joint Fictionalisation”. In: Bublitz, Wolfram/Lenk, Uta/Ventola, Eija (eds.) Coherence in Spoken and Written Discourse. Amsterdam/Philadelphia: John Benjamins: 125-150.

Kotthoff, Helga (2003): "Responding to Irony in Different Contexts: On Cognition in Conversation". Journal of Pragmatics 35/9: 1387-1411.

Kreuz, Roger (1996) “The Use of Verbal Irony: Cues and Constraints”. In: Mio, Jeffery/Katz, Albert (eds.): Metaphor: Implications and Applications. Mahwah, NJ, Erlbaum: 23-38. 
Kreuz, Roger/Link, Kristen (2002) "Asymmetries in the Use of Verbal Irony”. Journal of Language and Social Psychology 21/2: 127-143.

Kreuz, Roger/Roberts, Richard (1995) "Two Cues for Verbal Irony: Hyperbole and the Ironic Tone of Voice". Metaphor and Symbolic Activity 10/1: 21-31.

Krieger, Jörn (2002) Die Popularisierung des Medium Fernsehen. Vom Elite-Medium zum Schauplatz des Normalbürgers. Norderstedt: BoD.

Krüger, Udo (1998) „Thementrends in Talkshows der 90er Jahre. Talkshows bei ARD, ZDF, RTL, SAT.1 und PRO SIEBEN im Vergleich“. Media Perspektiven 12: 608-624.

Lapp, Edgar (1992): Linguistik der Ironie. Tübingen: Narr.

Lee, Seung-Hee/Tanaka, Hiroko (2016): “Affiliation and Alignment in Responding Actions". Journal of Pragmatics 100: 1-7.

Loosen, Wiebke (2016): „Das Leitfadeninterview - eine unterschätzte Methode“. In: AuerbeckLietz, Stefanie/Meyer, Michael (eds.): Handbuch nicht standardisierte Methoden in der Kommunikationswissenschaft. Wiesbaden, Springer: 139-155.

Mizzau, Marina (1984): L'ironia: la contraddizione consentita. Milano: Feltrinelli.

Morreal, John (1987): The Philosophy of Laughter and Humor. Albany NY: State University of New York Press.

Mortara Garavelli, Bice (2018): Manuale di retorica. Milano: Bompiani.

Mücke, Douglas (1978): “Irony Markers". Poetics 7: 399-350.

Norrick, Neal (1993): Conversational Joking: Humor in Everyday Talk. Bloomington: Indiana University Press.

Norrick, Neal (2009): “A Theory of Humor in Interaction”. Journal of Literary Theory 3/2: 261-284.

Nuolijärvi, Pirkko/Tiittula, Liisa (2011): "Irony in Political Television Debates". Journal of Pragmatics 43/2: 572-587.

Plake, Klaus (1999): Talkshows. Die Industrialisierung der Kommunikation. Darmstadt: Primus.

Prestin, Elke (2000): Ironie in den Printmedien. Wiesbaden: Deutscher Universitäts-Verlag.

Provine, Robert (2001): Laughter. A Scientific Investigation. New York: Penguin.

Quintilianus, Marcus Fabius: Institutionis Oratoriae/Ausbildung des Redners. Zwölf Bücher. Hrsg. und übersetzt von Helmut Rahn (1975). Zweiter Teil. Buch VII-XII. Darmstadt: Wissenschaftliche Buchgesellschaft.

Raskin, Victor (1985): Semantic Mechanisms of Humor. Dodrecht/Boston/Lancaster: Reidel.

Rockwell, Patricia (2001): "Facial Expression and Sarcasm". Perceptual and Motor Skills 93/1: 47-50.

Schneiderbauer, Christian (ed.) (2001): Daily Talkshows unter der Lupe. Wissenschaftliche Beiträge aus Forschung und Praxis. München: Reinhard Fischer.

Seto, Ken-Ichi (1998): “On Non-Echoic Irony”. In: Carston, Robyn/Uchida, Seiji (eds.) Relevance Theory: Applications and Implications. Amsterdam, Benjamins: 239-255.

Stivers, Tanya/Mondada, Lorenza/Steensig, Jakob (eds.) (2011): Knowledge and Affiliation in Social Interaction. The Morality of Knowledge in Conversation. Cambridge: Cambridge University Press. 
Tabacaru, Sabina/Lemmens, Maarten (2014): "Raised Eyebrows as Gestural Triggers in Humour: The Case of Sarcasm and Hyper-Understanding". The European Journal of Humour Research 2/2: 11-31.

Van Eimeren, Birgit/Gerhard, Heinz (1998). "Talkshows - Formate und Zuschauerstrukturen. Überblick über Entwicklung und Nutzung eines alltäglichen Programmformats". Media Perspektiven 12: 600-608.

Wiese, Heike (2012): Kiezdeutsch. Ein neuer Dialekt entsteht. München: C. H. Beck Verlag. Wilson, Deirdre/Sperber, Dan (1992): “On Verbal Irony”. Lingua 87/1: 53-76.

Yus, Francisco (1998): "Irony: Context Accessibility and Processing Effort". Pragmalingüística 5/6: 391-410. 